\title{
Molecular Typing of Uropathogenic Escherichia colli Strains Isolated from Patients by REP-PCR
}

DOI: $10.17691 /$ stm2017.9.3.09

Received December 14, 2016

Hakime Shokoohi Amiri, MSc Student';

Reza Ranjbar, PhD, Professor, Head;

Noshin Sohrabi, PhD, Assistant Professor, Department of Biology'; Faham Khamesipour, DVM, MPH, PhD, Researcher ${ }^{3,4}$;

${ }^{1}$ Payame Noor University, Tehran, Iran;

${ }^{2}$ Molecular Biology Research Center, Baqiyatallah University of Medical Sciences, Tehran, Iran;

${ }^{3}$ Health Policy Research Center, Institute of Health, Shiraz University of Medical Sciences, Shiraz, Iran;

${ }^{4}$ Cellular and Molecular Research Center, Sabzevar University of Medical Sciences, Sabzevar, Iran

The aim of the investigation was to determine the correlation between genetic patterns and strains of Escherichia coli (E. coli) and to evaluate the genetic diversity of $E$. coli strains isolated from patients admitted to Baqiyatallah Hospital, using the method of repetitive extragenic palindromic sequence polymerase chain reaction (REP-PCR).

Materials and Methods. A total of $100 \mathrm{E}$. coli strains collected from the samples of patients with urinary tract infections were studied. E. coli strains were identified and isolated by the standard bacteriological methods and their DNAs were extracted. The REP-PCR method with REP1R primer was used to type isolated strains.

Results. Ninety-eight out of $100 \mathrm{E}$. coli strains were typable and 2 strains were non-typable. Dendrogram showed that the isolated strains were differentiated by REP-PCR into 7 REP clusters (R1-R7) with $70 \%$ similarity. The highest number of strains was found in cluster R4 (22 strains) and this number was the lowest in cluster R3 (7 strains).

Conclusion. In Iran, the REP-PCR method has not been used for studying uropathogenic $E$. coli strains. This technique has shown high differential accuracy in our investigation. $E$. coli strains have been found to comprise various distinct clones which show the genetic diversity of bacteria in the patients studied.

Key words: uropathogenic E. coli; molecular typing; REP-PCR.

Urinary tract infections (UTI) are the most common bacterial infections which are characterized by bacterial colonization of the urinary system and often occur in women of all age groups. The strains of uropathogenic Escherichia coli (E. coli) are the most common pathogens isolated from UTI patients and often originate from the intestinal flora. The most common UTI symptom is dysuria which may be moderate or severe [1, 2]. In addition to common symptoms of the lower UTI, patients with the upper UTI or pyelonephritis may experience flank pain, fever, or nausea and vomiting [1]. E. coli is responsible for $80-90 \%$ of UTIs; the remaining UTIs are caused by Staphylococcus saprophyticus, Citrobacter, Klebsiella, and Enterobacter [3]. E. coli produces purple colonies on MacConkey agar culture medium because it is a lactose-positive bacterium which ferments sugar and produces acid. The same process also occurs in EMB culture medium (eosin and methylene blue agar) where the colonies turn dark purple with greenish metallic sheen. Commensal species of $E$. coli of normal intestinal flora are usually harmless for the host and cause disease only in immunocompromised hosts. However, some species of $E$. coli are highly potential primary pathogens and cause disease after acquisition of certain virulent characteristics [4].

In various human diseases induced by $E$. coli, pathogenic $E$. coli is generally divided into two main categories: diarrheagenic $E$. coli and extraintestinal pathogenic $E$. coli which have two major pathotypes, uropathogenic $E$. coli and neonatal meningitis $E$. coli. Extraintestinal bacteria can colonize different sites and cause various infections outside the gastrointestinal tract, with the urinary tract being most commonly affected $[5,6]$. Uropathogenic $E$. coli encode different adhesive structures (adhesin) called pili and fimbriae, such as F1C, S, P, and $\mathrm{Dr}$ adhesion family [7]. Fimbriae play an important role in bacteria binding to mucosal sites of the digestive tract, the vagina, and the urethra. Other factors, such as bacterial lipopolysaccharides, capsules, hemolysins, and siderophores, help the virulence of uropathogenic E. coli [8-10]. A strain of uropathogenic E. coli can comprise several different pili and change one type of pilus to another type depending on the changes in the environment. On reaching the urinary tract, uropathogenic E. coli first of all binds to the host epithelial cells using pili and fimbriae not to be cleared up by urine flow [11].

For contacts: Reza Ranjbar, e-mail: ranjbarre@gmail.com 
Molecular typing can be used to identify nosocomial infections and infection reservoirs and also to isolate specific genotypes in conjugation with a specific bacterium. Besides, it gives us more knowledge about the principles of epidemiology, evolution, and spread of many bacterial diseases [12-15]. Repetitive extragenic palindromic (REP) sequence originally detected in Salmonella typhimurium and $E$. coli is a 39 bp repetitive sequence that may occur up to 282 times in $E$. coli genome. These sequences seem to regulate expression of genes through controlling mRNA stability [16]. The REP-PCR (repetitive extragenic palindromic sequence polymerase chain reaction) method is based on the fact that the areas located between repeated segments have different sizes and therefore create different patterns during PCR and electrophoresis, so the strains can be easily distinguished and recognized by comparing these patterns [17].

A certain advantage of this method is that there is no need to know the target sequence for synthesis of primers in most bacteria. Given high reproducibility and distinguishability of this technique, it has recently been considered an appropriate method for epidemiological studies of most bacteria along with pulsed field gel electrophoresis. Therefore, the REPPCR molecular technique is a commercial PCR-based, semi-automatic and fast typing system that can be easily implemented by a molecular technologist with some experience [18].

One of the most important benefits of REP-PCR is that the method can be used in a large number of gramnegative and some gram-positive bacteria, without any information about the bacterial genome [19]. In addition, it is a reliable technique compared to vague and timeconsuming phenotypic and biochemical methods. This method has the advantages of the ease of use, speed, high distinction power, and applicability in all laboratories. REP-PCR can be used with biomarkers such as (GTG)5, REP1R-I, and REP2R-I [20].

The aim of the investigation was to determine molecular types of uropathogenic $E$. coli as one of the main causes of urinary tract infections using the REPPCR technique with REP1R primer.

Materials and Methods. This cross-sectional study was carried out in 2014 and involved the patients with UTI admitted to Tehran Baghiatallah Hospital. The study complies with the Declaration of Helsinki (the Declaration was passed in Helsinki, Finland, June 1964, and revised in October 2000, Edinburgh, Scotland) and was performed following approval by the Ethic Committee of Baqiyatallah University of Medical Sciences. Written informed consent was obtained from every patient.

The samples were cultured in chrome agar culture medium and incubated for $24 \mathrm{~h}$. If purple colonies grew, the presence of $E$. coli was confirmed by transferring and incubating the colonies in EMB agar medium at $37^{\circ} \mathrm{C}$ for $18 \mathrm{~h}$. Metallic green colonies indicated the presence of $E$. coli and underwent further analysis. All colonies cultured in EMB medium were transferred to the medium enriched by Luria-Bertani broth and cultured in a shaking incubator for 18 to $24 \mathrm{~h}$. Afterwards, DNA was purified using boiling extraction method. The obtained genomic solution could be used directly in PCR or stored at $4^{\circ} \mathrm{C}$ for subsequent tests. For long-term storage, the extracted solution must be kept at $20^{\circ} \mathrm{C}$ because DNA may be subjected to acid hydrolysis.

After testing the purity of DNA by NanoDrop analyzer (Thermo Fisher Scientific, USA) and confirming its suitability for performing PCR, the extracted DNA was prepared for PCR. The reaction mixture contained $1 \mu \mathrm{l}$ template DNA, $2 \mu \mathrm{l}$ REP1R primer, $1 \mu \mathrm{l} \mathrm{MgCl}_{2}, 6 \mathrm{ml}$ deionized distilled water, and $10 \mu \mathrm{l}$ master mix in a total volume of $20 \mathrm{ml}$. PCR amplification was performed in an Eppendorf gradient thermal cycler (Eppendorf, Germany) with an initial denaturation at $95^{\circ} \mathrm{C}$ for $7 \mathrm{~min}$, followed by 32 cycles consisting of denaturation ( $1 \mathrm{~min}$ at $\left.95^{\circ} \mathrm{C}\right)$, annealing $\left(1 \mathrm{~min}\right.$ at $\left.41^{\circ} \mathrm{C}\right)$, extension $(3 \mathrm{~min}$ at $65^{\circ} \mathrm{C}$ ) and the phase of final extension at $65^{\circ} \mathrm{C}$ for $16 \mathrm{~min}$. To visualize the products of PCR amplification, $5 \mu \mathrm{l}$ of the final solution was mixed with $1 \mu \mathrm{l}$ of loading buffer and electrophoresed on $1.5 \%$ agarose gel at a voltage of $90 \mathrm{~V}$ for $70 \mathrm{~min}$. The $100 \mathrm{bp}$ DNA ladder was used as a molecular standard to verify the particular REP segments. The gel was stained with ethidium bromide for 12 min and the segments became visible with UV light. Finally, REP-PCR bands were expressed as 0 and 1 to denote the absence and presence of the bands, respectively. A matrix of numbers zero and one was prepared in Excel and the relevant dendrogram was drawn using InSilico website.

Results. A total of 100 E. coli strains isolated from patients with UTI were evaluated in this study, with 98 out of 100 strains being typable and 2 strains nontypable. Seventeen bands ranging from 100 to 3,100 bp were amplified in different profiles (See Figure).

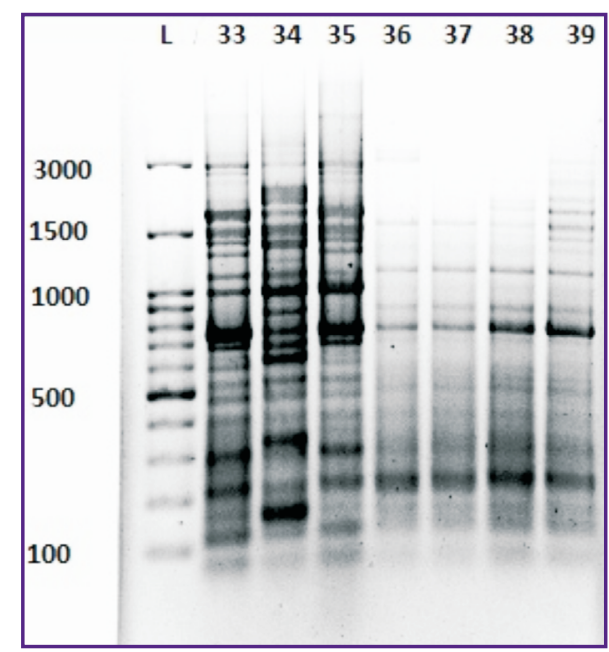

Profile of PCR products of some samples, column $L$ is the size of the marker 
Table 1

The mean age and percentage of females in each REP cluster

\begin{tabular}{llllllll}
\hline \multicolumn{1}{c}{ Parameters } & \multicolumn{10}{c}{ Cluster } \\
\cline { 2 - 9 } & R1 & R2 & R3 & R4 & R5 & R6 & R7 \\
\hline Mean age (years) & 45 & 58 & 39 & 49 & 38 & 96 & 45 \\
\hline Percentage of females (\%) & 75 & 75 & 85 & 72 & 90 & 81 & 70 \\
\hline
\end{tabular}

Dendrogram analysis showed that the isolates were grouped by REP-PCR in 7 REP clusters (R1-R7) with $70 \%$ similarity. Out of 100 studied patients with UTI, 79 were women and 21 were men and the prevalence of the disease had almost the same slope in different age groups indicating the lack of relationship between the age and risk of the disease. It should be noted that the analysis of the proportion of women and the mean age in terms of strains showed that R5 had the highest percentage of women and the lowest mean age among the clusters (Table 1).

Discussion. Uropathogenic E. coli are predominant bacteria causing UTI, especially in women. UTI is one of the most common human infections influenced by gender and age [2, 21]. REP-PCR reveals DNA patterns and in fact allows differentiation between bacterial species [22-23] making it a useful technique for typing various bacteria [23]. Non-coding dispersed repetitive sequences have been observed preferably in gramnegative bacteria [24]. Previous studies have identified the presence of repetitive DNA elements in E. coli using REP-PCR. Four cases of UTI out of 100 samples under study were observed in female newborns during this investigation. In fact, UTI occurs in 0.1 of newborns. In the first two months of life, UTI is more common in men, but after the second month until adulthood, UTI is more common in females. However, in 100 samples studied, none of the babies with UTI were male. Five cases of infection were seen in girls aged one to ten years due to their short urethra, failure to maintain personal hygiene and urine retaining in children. In boys, circumcision reduces the risk of UTI. This risk also declines in adolescence. In our study, only two cases of infection were observed in adolescent girls. UTI increases severely in young women due to sexual relationships, pregnancy or contraceptives. In this study, 22 cases of UTI were observed in the age range of 20 to 25 years. After menopause, UTI incidence may increase due to estrogen deficiency.

Given the information on DNA polymorphism, we investigated polymorphism of $E$. coli strains using REP1R primer which is adequately powerful for differentiating highly similar polymorphisms. As shown by the dendrogram, the isolates were categorized with high accuracy into 7 groups with genomic profile similarity of $70 \%$. According to clustering performed, the highest and the lowest number of strains were seen in clusters R4 (22 strains) and R3 (7 strains), respectively. Cluster R4 included 22 strains and was the largest cluster of isolates, with strains 65 and 95, 57 and 60, 58 and 59 having genomic profile similarity of more than $70 \%$. Strains 65 and 95 in cluster R4 had similar genomic profiles and belonged to the same person, the samples being harvested in two different time periods, which indicated a recurrent infection caused by the strain. These results demonstrate the accuracy of the method. In cluster R5 (21 strains), strains 73 and 74 had genomic profile similarity of more than $70 \%$, strains $41,43,44,45$, and 46 in cluster R7 (17 strains) also had similarity of more than $70 \%$ (Table 2). Clusters R1 (12 strains), R2 (8 strains), R3 (7 strains) as the least populated clusters and cluster R6 (11 strains) had no similarity in their genomic profiles.

Band 100 was the most frequent band and was observed in $90 \%$ of strains, while bands 700 and 800 were alternatively seen among the isolates. It is noteworthy that the analysis of the proportion of females and the mean age in terms of strains showed that among the clusters, R5 had the highest percentage of females and the lowest mean age. Comparison of the antibiogram data with the dendrogram data provided remarkable results. All samples in cluster R2 were resistant to ampicillin and sensitive to nitrofurantoin, all samples in cluster R3 were sensitive to nitrofurantoin, the majority of samples in cluster R5 were sensitive or semi-sensitive to amikacin and all samples in cluster R6 were sensitive to nitrofurantoin.

Due to high UTI incidence, identifying antibiotic sensitivity of bacteria which reflects the relative resistance of disease-producing bacteria to the commonly used antibiotics seems necessary in the present study. Using antibiotics correctly and identifying the resistance of bacteria isolated from UTI patients may play an important role in controlling the disease and its dangerous consequences. The REP-PCR method has already been used for evaluation of $E$. coli resistance to antibiotics. For example, Rashid et al. [25] have used this method to study drug resistance of extended spectrum beta-lactamase and identify extended spectrum betalactamase producers at the genotype level. This method has also been widely used for diagnostic purposes. For example, in May 2003, McLellan et al. studied E. coli isolated from common sources of fecal contamination and genetic relations of strains in each host group using the REP-PCR molecular method [26]. The study showed that the REP-PCR molecular method was more accurate and efficient than DNA fingerprint of fecal $E$. coli in animal or human hosts. Many other foreign studies

Table 2

The number of strains in each cluster

\begin{tabular}{lccccccc}
\hline \multirow{1}{*}{ Number } & \multicolumn{8}{c}{ Cluster } \\
\cline { 2 - 8 } & R1 & R2 & R3 & R4 & R5 & R6 & R7 \\
\hline Absolute number & 12 & 8 & 7 & 22 & 21 & 11 & 17 \\
\hline Percentage & 12.2 & 8.16 & 7.4 & 22.4 & 21.4 & 11.22 & 17.3 \\
\hline
\end{tabular}


devoted to different $E$. coli strains have been performed using this method. A limited number of similar studies have been done in Iran. UTI is a common problem among the female population and further studies with a larger sample size are required to solve it effectively, with the role of other factors involved in the disease development being taken into account.

Conclusion. The results of this study have shown that REP-PCR is a reliable method for classification, molecular genotyping, and identification of phylogenetic relationships between very close bacterial species and even distinct species. The use of this powerful method has provided the possibility to determine with high precision that $E$. coli bacteria studied in this investigation comprise various distinct clones which indicate the diversity of bacteria in the studied patients.

Contributions of the authors to the study. All the authors have equally contributed to this work. All the authors have read and approved of the final manuscript.

Conflicts of Interest. The authors have no conflicts of interest to disclose.

\section{References}

1. Lane D.R., Takhar S.S. Diagnosis and management of urinary tract infection and pyelonephritis. Emerg Med Clin North Am 2011; 29(3): 539-552, https://doi.org/10.1016/j. emc.2011.04.001.

2. Tajbakhsh E., Tajbakhsh S., Khamesipour F. Isolation and molecular detection of gram negative bacteria causing urinary tract infection in patients referred to Shahrekord Hospitals of Iran. Iran Red Crescent Med J 2015; 17(5): 17(5): e24779, https://doi.org/10.5812/ircmj.17(5)2015.24779.

3. Mead P.S., Slutsker L., Dietz V., McCaig L.F., Bresee J.S., Shapiro C., Griffin P.M., Tauxe R.V. Food-related illness and death in the United States. Emerg Infect Dis 1999; 5(5): 607-625, https://doi.org/10.3201/eid0505.990502.

4. Tajbakhsh E., Khamesipour F., Ranjbar R., Ugwu I.C. Prevalence of class 1 and 2 integrons in multi-drug resistant Escherichia coli isolated from aquaculture water in Chaharmahal Va Bakhtiari province, Iran. Ann Clin Microbiol Antimicrob 2015; 14(1): 37, https://doi.org/10.1186/s12941-015-0096-y.

5. Johnson J.R., Russo T.A. Extraintestinal pathogenic Escherichia coli: "the other bad E. coli". J Lab Clin Med 2002; 139(3): 155-162, https://doi.org/10.1067/mlc.2002.121550.

6. Ranjbar R., Pezeshknejad P., Khamesipour F., Amini K., Kheiri R. Genomic fingerprints of Escherichia coli strains isolated from surface water in Alborz province, Iran. BMC Research Notes 2017; 10(1): 295, https://doi.org/10.1186/ s13104-017-2575-z.

7. Snyder J.A., Haugen B.J., Lockatell C.V., Maroncle N., Hagan E.C., Johnson D.E., Welch R.A., Mobley H.L. Coordinate expression of fimbriae in uropathogenic Escherichia coli. Infect Immun 2005; 73(11): 7588-7596, https://doi.org/10.1128/iai.73.11.7588-7596.2005.

8. Svanborg C., Godaly G. Bacterial virulence in urinary tract infection. Infect Dis Clin North Am 1997; 11(3): 513-529, https://doi.org/10.1016/s0891-5520(05)70371-8.

9. Donnenberg M.S., Welch R.A. Virulence determinants of uropathogenic Escherichia coli. In: Mobley H.L.T., Warren J.W. (editors). Urinary tract infections: molecular pathogenesis and clinical management. Washington, DC, USA: ASM Press; 1996; p. 135-174.

10. Foxman B., Zhang L., Palin K., Tallman P., Marrs C.F. Bacterial virulence characteristics of Escherichia coli isolates from first-time urinary tract infection. $J$ Infect Dis 1995; 171(6): 1514-1521, https://doi.org/10.1093/infdis/171.6.1514.

11. Dodson K.W., Pinkner J.S., Rose T., Magnusson G., Hultgren S.J., Waksman G. Structural basis of the interaction of the pyelonephritic $\mathrm{E}$. coli adhesin to its human kidney receptor. Cell 2001; 105(6): 733-743, https://doi.org/10.1016/ s0092-8674(01)00388-9.

12. Li W., Raoult D., Fournier P.-E. Bacterial strain typing in the genomic era. FEMS Microbiol Rev 2009; 33(5): 892-916, https://doi.org/10.1111/j.1574-6976.2009.00182.x.

13. Rahimi E., Khamesipour F., Yazdi F., Momtaz H. Isolation and characterization of enterohaemorragic Escherichia coli 0157:H7 and EHEC 0157:NM from raw bovine, camel, water buffalo, caprine and ovine milk in Iran. Kafkas Univ Vet Fak Derg 2012; 18: 559-564, https://doi. org/10.9775/kvfd.2011.5738.

14. Hemmatinezhad B., Khamesipour F., Mohammadi M., Safarpoor Dehkordi F., Mashak Z. Microbiological investigation of O-serogroups, virulence factors and antimicrobial resistance properties of shiga toxin-producing Escherichia coli isolated from ostrich, turkey and quail meats. J Food Saf 2015; 35(4): 491-500, https://doi.org/10.1111/jfs.12199.

15. Ranjbar R., Hosseini S., Zahraei-Salehi T., Kheiri R., Khamesipour F. Investigation on prevalence of Escherichia coli strains carrying virulence genes ipaH, est $A$, eaeA and bfpA isolated from different water sources. Asian Pac J Trop Dis 2016; 6(4): 278-283, https://doi.org/10.1016/s22221808(15)61031-3.

16. Versalovic J., Lupski J.R. Interspersed repetitive sequences in bacterial genomes. In: de Bruijn F.J., Lupski J.R. Weinstock G.M. (editors). Bacterial genomes. Springer US; 1998; p. 38-48, https://doi.org/10.1007/978-1-4615-6369-3_5.

17. Molecular typing in bacterial infections. de Filippis I. McKee M.L. (editors). Humana Press; 2013, https://doi. org/10.1007/978-1-62703-185-1.

18. Pitout J.D., Campbell L., Church D.L., Wang P.W., Guttman D.S., Gregson D.B. Using a commercial diversilab semiautomated repetitive sequence-based PCR typing technique for identification of Escherichia coli clone ST131 producing CTX-M-15. J Clin Microbiol 2009; 47(4): 1212-1215, https://doi.org/10.1128/jcm.02265-08.

19. Rademaker J.L.W., Louws F.J., de Bruijn F.J. Characterization of the diversity of ecologically important microbes by rep-PCR genomic fingerprinting. In: Akkermans A.D.L., van Elsas J.D., de Bruijn F.J. (editors). Molecular microbial ecology manual. Suppl. 3, Chap. 343. Dordrecht: Kluwer Academic Publishers; 1998; p. 1-26.

20. Tafvizi F., Tajabadi Ebrahimi M. Application of repetitive extragenic palindromic elements based on PCR in detection of genetic relationship of lactic acid bacteria species isolated from traditional fermented food products. J Agr Sci Tech 2015; 17: 87-98.

21. Rosen D.A., Hooton T.M., Stamm W.E., Humphrey P.A., Hultgren S.J. Detection of intracellular bacterial communities in human urinary tract infection. PLoS Med 2007; 4(12): e329, https://doi.org/10.1371/journal.pmed.0040329.

22. Olive D.M., Bean P. Principles and applications of methods for DNA-based typing of microbial organisms. J Clin Microbiol 1999; 37(6): 1661-1669. 
23. Elias W.P., Uber A.P., Tomita S.K., Trabulsi L.R., Gomes T.A. Combinations of putative virulence markers in typical and variant enteroaggregative Escherichia coli strains from children with and without diarrhoea. Epidemiol Infect 2002; 129(1): 49-55, https://doi.org/10.1017/s0950268802007136.

24. Woods C.R., Versalovic J., Koeuth T., Lupski J.R. Whole-cell repetitive element sequence-based polymerase chain reaction allows rapid assessment of clonal relationships of bacterial isolates. J Clin Microbiol 1993; 31(7): 1927-1931.
25. Rashid M., Rakib M.M., Hasan B. Antimicrobial-resistant and ESBL-producing Escherichia coli in different ecological niches in Bangladesh. Infect Ecol Epidemiol 2015; 5: 26712 , https://doi.org/10.3402/iee.v5.26712.

26. McLellan S.L., Daniels A.D., Salmore A.K. Genetic characterization of Escherichia coli populations from host sources of fecal pollution by using DNA Fingerprinting. Appl Environ Microbiol 2003; 69(5): 2587-2594, https://doi. org/10.1128/aem.69.5.2587-2594.2003. 